\title{
Friction behaviour of hydrophilic lubricious coatings for medical device applications
}

Agata Niemczyk",a, Miroslawa El Fraya, Steve E. Franklin ${ }^{b}$

${ }^{a}$ West Pomeranian University of Technology, Szczecin, Biomaterials and Microbiological Technologies, Al. Piastow 45, 71-311 Szczecin, Poland

bPhilips Research, Eindhoven, The Netherlands

*Corresponding author

e-mail addresses: aniemczyk@zut.edu.pl (A. Niemczyk)

mirfray@zut.edu.pl (M. El Fray)

s.e.franklin@philips.com (S. Franklin)

\begin{abstract}
The friction behaviour of new chitosan derivative coatings obtained by chemical modification of chitosan with fatty acids (linoleic and dilinoleic acid) has been investigated in order to explore their potential as endovascular catheter coatings and to benchmark them against commercially available coatings used in endovascular catheter applications. An in vitro tribological system was developed that was intended to represent to a limited extent the in vivo tribological conditions of a typical endovascular catheterization procedure. Continuous reciprocating sliding tests were carried out with uncoated and coated polymer specimens. The results showed that all of the coatings tested decreased the coefficient of friction compared to the uncoated polymer. Compared to a neat chitosan coating, the chitosan derivative coatings showed a clear reduction in the coefficient of friction to levels similar to those of the commercially-available coatings. A comparison between the friction results and contact angle measurements carried out on the coatings indicated that a range of contact angle values exists for which the friction coefficient is at a minimum. The reason for this is unclear and further studies are required in order to confirm and investigate the trend, especially within the context of hydrophilic lubricious coating development.
\end{abstract}

Key words: friction coefficient, chitosan coatings, hydrogels, catheter coatings

\section{Introduction}

Rapid progress and increasing needs in the biomedical field have contributed to the extensive development of polymeric coatings. Many of the improvements of currently used devices and new solutions have been led by the variety and tailor-made properties of polymer coatings [1-5]. The application of hydrophilic-type coatings can provide advantages such as good wetting, inducement of capillary flow, good biocompatibility, low protein adsorption, and in contact with blood, reduction of the risk of thrombogenesis. In endovascular catheterisation procedures, friction against the luminal surface of a blood vessel along which the catheter passes is integral to the insertion, manipulation and removal of the catheter by the operator. A hydrophilic coating on the catheter can replace or supplement the lubricating action of the endothelial surface layer[6], facilitating catheterisation[7]. Existing hydrophilic coatings are formed by polymers with functional groups able to absorb water molecules, such as amino, hydroxyl or carboxyl groups. For this reason the most commonly used polymers are acrylic polymers, polyvinyl alcohol (PVA), polyvinyl pyrrolidone (PVP), polyethylene glycols (PEG)s, some natural polymers such as polysaccharides, and various derivatives and their copolymers [8]. The primary focus of commercially available hydrophilic coatings for endovascular catheters is on improving the lubricity, maintaining a low and stable friction for the relatively short distance that the catheter slides against the vessel wall during the catheterisation procedure. This distance is associated with inserting, positioning, and removing the catheter after the treatment has taken place. For such applications, provided the friction does not increase during sliding, the coating wear behaviour is of minor importance since the catheters are single-use devices, discarded after use. 
However, while these coatings have proven effective at reducing the incidence of spasm and improving patient comfort, the use of hydrophilic sheaths does not appear to prevent the reduction in flow mediated dilation, an index of endothelial dysfunction[9], and did not markedly influence the incidence of radial occlusion[10,11]. Further, the use of certain hydrophilic coatings (for example, those manufactured by $\operatorname{Cook}[12,13])$ has been associated with the formation of sterile granulomas and abscesses[14-18]. Clearly, there is substantial room for improvement in the field of lubricious coatings for endovascular catheter use and, in particular, biodegradability would be a marked advantage.

Chitosan is a natural hydrophilic polysaccharide, which can absorb water, due to the presence of amino groups in the structure, despite being insoluble in water. It is also non-toxic, biocompatible, and, importantly, biodegradable, in addition to possessing some antimicrobial activity [19-21]. To improve the physical and biological properties, as well as widen the range of potential applications, versatile chitosan derivatives have been synthesised [22]. Amphiphilic derivatives are especially interesting, due to the presence of a hydrophobic molecules in the chitosan structure, which can modify the rheological behaviour, resulting in self-assembly ability [23-25]. There are also studies that show improvements in blood compatibility and antimicrobial activity [26]. Our prior work has explored the modification of chitosan with fatty acids (linoleic and dilinoleic acid) and examined the antimicrobial properties of these new materials $[27,28]$. Catheter related infections remain a serious problem in both intravascular applications[29] (indwelling catheters, in particular), as well as in urinary applications.[30] As a result, the combination of blood compatibility and antimicrobial properties, if combined with good lubricity, would make these chitosan derivatives of great interest as coatings for catheter applications.

The aim of our present work was to investigate the friction behaviour of the chitosan fatty acid derivative coatings, benchmarking them against commercially available coatings, in order to assess their potential for catheter coating applications. For this purpose an in vitro biotribology test system was developed. It was our goal to emulate the in vivo friction conditions of a typical endovascular catheterization procedure, whilst avoiding many of the experimental and regulatory issues associated with ex vivo and in vivo testing. The test system, which was necessarily limited in its ability to simulate the real conditions, consisted of synthetic materials instead of living tissues or blood to minimize variability resulting from the lack of reproducibility of living tissues and also to facilitate comparisons between the coatings.

\section{Experimental}

\subsection{Tribological system}

An in vitro tribological system was developed in order to compare the friction behaviour of hydrophilic coatings intended to be used on the outside of cardiovascular catheters. As far as possible, the individual elements of the system were matched to reflect the conditions of the catheterization process.

\subsubsection{Tribological testing conditions}

Reciprocating sliding friction tests were carried out using a CETR (Brüker) UMT Multi-Specimen Test System. The test set-up is shown schematically in Fig.1. In line with the application, the value and stability of the friction coefficient was investigated for a total sliding distance of $0.9 \mathrm{~m}$, this being a distance considered to be typical of a routine endovascular catheterisation procedure. Because it is the friction behaviour rather than the wear behaviour that determines the performance in this application, the actual wear of the coatings in terms of volumetric wear loss was not investigated here. The coatings to be tested were deposited on injection-moulded spherical poly(ether-bamide)(PA) polymer probes (representing the catheter material) with a diameter of $3.95 \mathrm{~mm}$ and rubbed against flat pieces of soft poly(vinyl alcohol)(PVA) hydrogel which represented the blood 
vessel. During the test, the probes were partially immersed to a depth of approximately $3 \mathrm{~mm}$ in a glycerol-water mixture representing human blood. A specially-designed holder enabled the wet hydrogel to be fixed in place during the test, see Fig. 2. All friction tests were carried out at a load 0.1 $\mathrm{N}$, velocity $0.5 \mathrm{~mm} / \mathrm{s}$ and stroke $10 \mathrm{~mm}$ at room temperature and each test was repeated at least five times using fresh materials and coatings for each test to obtain the average and standard deviation values shown in the results section. Estimates of the contact radius ( $r$ ) of the circular contact area and of the mean contact pressure $\left(P_{m}\right)$ were made using a Hertzian contact model (see below) where $R$ is the radius of the spherical probe, $\mathrm{N}$ is the normal force and $\mathrm{E}^{\prime}$ is the effective modulus of elasticity:

(Spherical)contact Radius $(r)=\left(3 R N / 2 E^{v}\right)^{1 / 3}$

Mean contact pressure $P_{m}=\frac{W}{\pi \cdot r^{m}}=\frac{1}{\pi} \cdot\left(\frac{2 \cdot E^{r}}{3 \cdot R}\right)^{2 / 3} \cdot N^{1 / 3}$

The effective elastic modulus is given by:

Effective elastic modulus $E^{0-1}=\frac{1}{2}\left(\frac{1-v_{P}}{E_{P}}+\frac{1-v_{H}}{E_{H}}\right)$

Where $E_{P}, E_{H}, U_{P}$ and $U_{H}$ are the elastic moduli and Poisson constants of the probe and hydrogel respectively.

Manufacturer's data (Arkema Group) was used for the elastic modulus of the PA33 material: 14.6 MPa. For the harder PA69 material the manufacturer's data (Arkema Group) on the flexural modulus was used as a first approximation of the elastic modulus: $510 \mathrm{MPa}$ (ISO 178 test method). This is a reasonable assumption in view of the small expected deformation of the polymer in this case. For the PVA hydrogel the value of the compressive modulus at $0.1 \mathrm{~N}$ force was used as a first approximation of the elastic modulus: $16.8 \mathrm{kPa}$. A value of 0.4 was taken as the Poisson constant for the probe polymers PA33 and PA69 and a value of 0.44 was used for the PVA hydrogel[31]. Using the above equations and values, the estimated mean contact pressure in our experiments was $0.01 \mathrm{MPa}$ for both the PA33 and PA69 material.

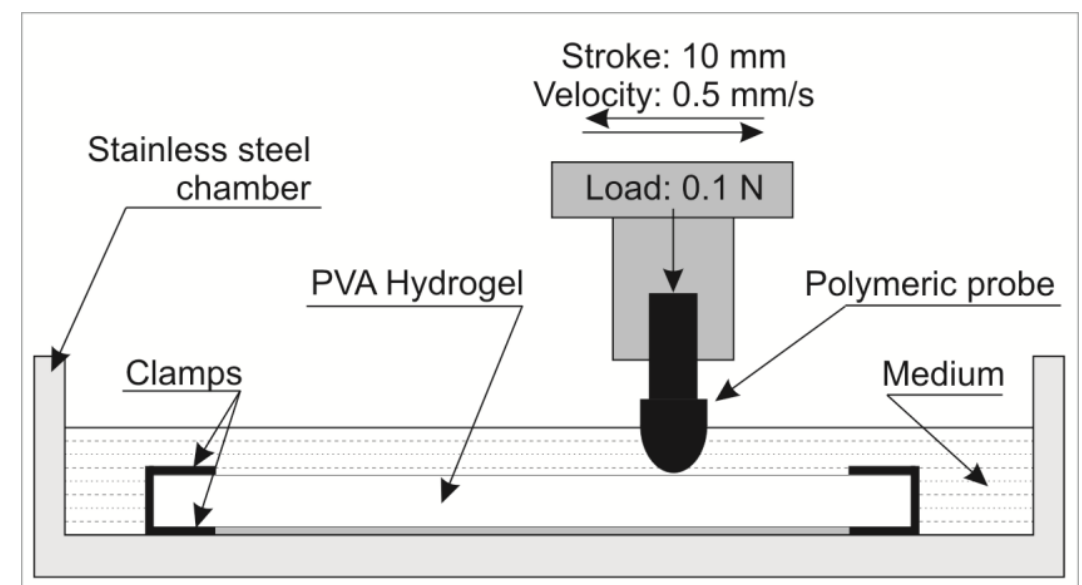

Fig. 1 Schematic diagram of the tribosystem. 


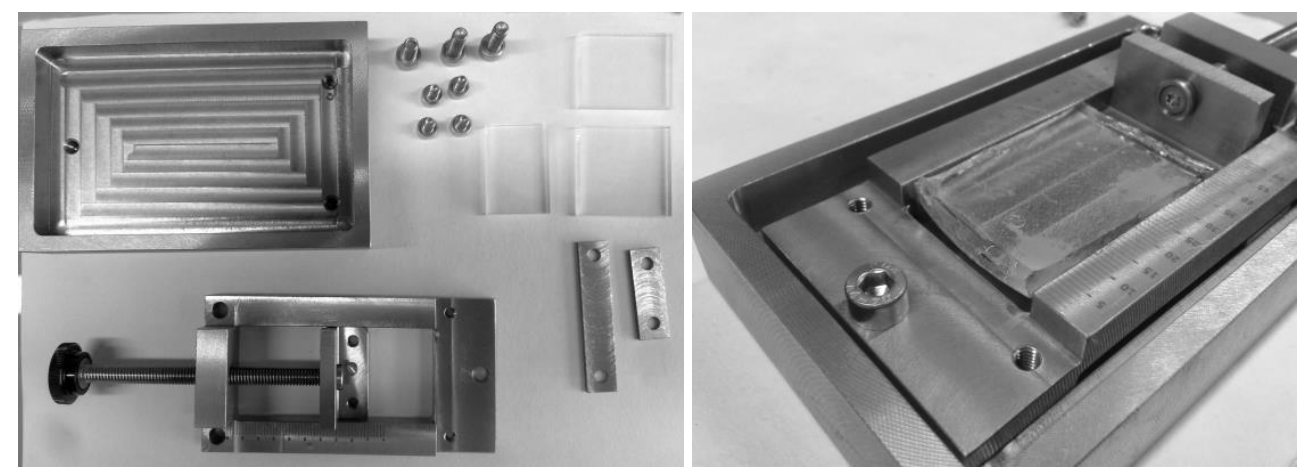

Fig. 2 Hydrogel fixing tool used in the tribological tests.

\subsubsection{Blood Vessel analogue (PVA Hydrogel)}

A key element of the tribological system developed here was the selection of contact partner of the coatings intended to simulate the blood vessel lumen. It is apparent from previous research into biomechanical modelling and the development of synthetic blood vessels for clinicians' training devices that PVA hydrogel can be used to simulate the interior of blood vessels [32-34]. In addition to having similar mechanical properties, PVA hydrogels have a high water content resulting in a low surface friction resistance and low interfacial energy with water or biological fluids [35], thus these materials canmimic human soft tissues.

An important advantage of PVA is the possibility to plan/design its properties by suitable selection of the ratio of hydrogel components, for example by changing the PVA and/or crosslinking agent concentration [36-38]. In this study, based on the work of Watler et al. [36], $10 \mathrm{wt} \%$ of PVA was chemically cross-linked using glutaraldehyde in the presence of magnesium chloride. The reaction of glutaraldehyde with hydroxyl groups of PVA results in a stable chemical network of acetal bridges. In terms of simulation of the blood vessel for tribological testing the crucial feature was the low friction and reproducible friction behaviour of the PVA surface.

The hydrogel was produced from PVA powder, trade name Elvanol 90-50 from DuPont (degree of hydrolysis and molecular masses: 99 to $99.8 \%$ and $M_{n}=29700 \mathrm{~g} / \mathrm{mol}, \mathrm{M}_{\mathrm{w}}=55800 \mathrm{~g} / \mathrm{mol}$ ).

Glutaraldehyde solution (GA) and magnesium chloride were used for the chemical crosslinking of the PVA. To produce the PVA hydrogel, PVA powder ( $10 \%$ wt.) was fully dissolved in distilled water for 48 hours at $70^{\circ} \mathrm{C}$. The PVA solution was subsequently cooled and $\mathrm{MgCl}_{2}$ ( $2 \%$ wt.) and $\mathrm{GA}$ ( $4 \%$ wt.) were added. This mixture was then vigorously mixed for $5 \mathrm{~min}$ and cast into plastic petri-dishes. Drying was carried out very slowly ( $48 \mathrm{~h}$ ) to avoid the formation of surface irregularities as a result of shrinkage. Subsequently, hydrogel cross-linking was carried out for $2 \mathrm{~h}$ at $70^{\circ} \mathrm{C}$. Once hydrated the water content of the final material was $90 \%$.

\subsubsection{Blood analogue (Glycerol water mixture)}

According to the literature, a common substance that has been used as a human blood analogue is a glycerol solution [39-41] and it was chosen as the blood analogue for the tribological system reported here. To obtain a fluid with room-temperature viscosity within the range of human blood viscosity at body temperature (around $3-4 \times 10^{-3} \mathrm{~Pa} \cdot \mathrm{s}[42,43]$ ), a $46 \%$ weight ratio of glycerol to water was used.

\subsubsection{Polymeric material used to represent the catheter}

Polymeric probes were made by injection moulding a poly(ether-b-amide) (PA, trade name Pebax ${ }^{\circledR}$ ), a commonly used polymer in catheters. Typically, guide catheters are constructed from several hardnesses of polymers, see Fig. 3. This is to provide flexibility at the distal end of the catheter to allow easier negotiation of bends. The catheter tip is typically the softest material so that it is relatively atraumatic. The material further away from the tip is relatively stiff in order that the catheter has sufficient back-up. For this reason, two PAs with different hardness, 33 Shore D (Pebax ${ }^{\circledR}$ 3533) and 69 Shore D (Pebax ${ }^{\circledR} 7233$ ) were tested (and marked in this work as PA33, and PA69, respectively), to reflect the different degrees of flexibility in various parts of the catheter. To make the 
tested probes resemble the catheter material more closely, 3\% blue dye (MEVOPUR ${ }^{\circledR}$ AH5M176002 medical colour master batch from Clariant International Ltd., Switzerland) was added to the polymer granulate during injection moulding of the probes, see Fig 4.

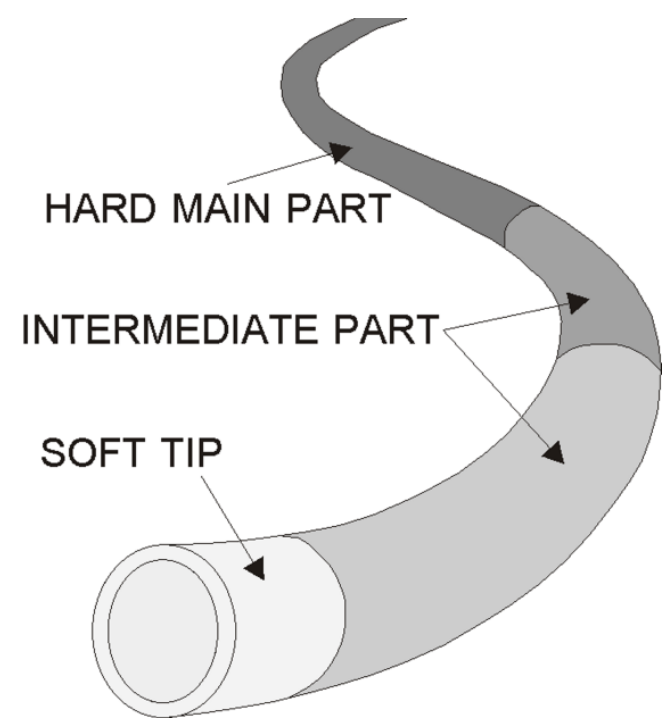

Fig. 3. The different degrees of flexibility in various parts of the catheters.

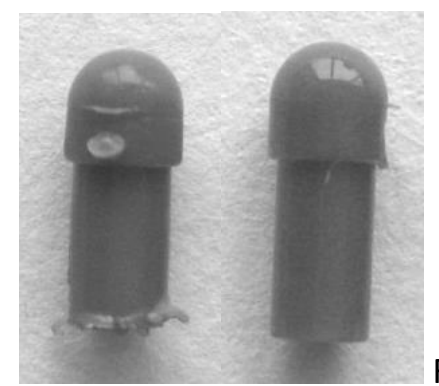

right: 69 ShD.

Fig. 4 Poly(ether-b-amide)(PA) probes, injection moulded. Left: 33 ShD,

\subsubsection{Coatings}

\subsubsection{Chitosan Derivative Coatings}

Coatings of chitosan derivatives were obtained by chemical modification of chitosan (CH) (Chitosan low molecular weight, Sigma-Aldrich) with fatty acids. Linoleic acid (LA) (Sigma-Aldrich) and dilinoleic acid (DLA) (Croda, The Netherlands), which are natural hydrophobic compounds, were intended to improve the physical and biological properties of chitosan. Details of this chemical alteration and characterization of antibacterial properties of these hydrophobic chitosan derivatives, CHLA and CHDLA, are described elsewhere $[27,28]$. The chemical structures of the chitosan derivatives tested are shown in Fig. 5.

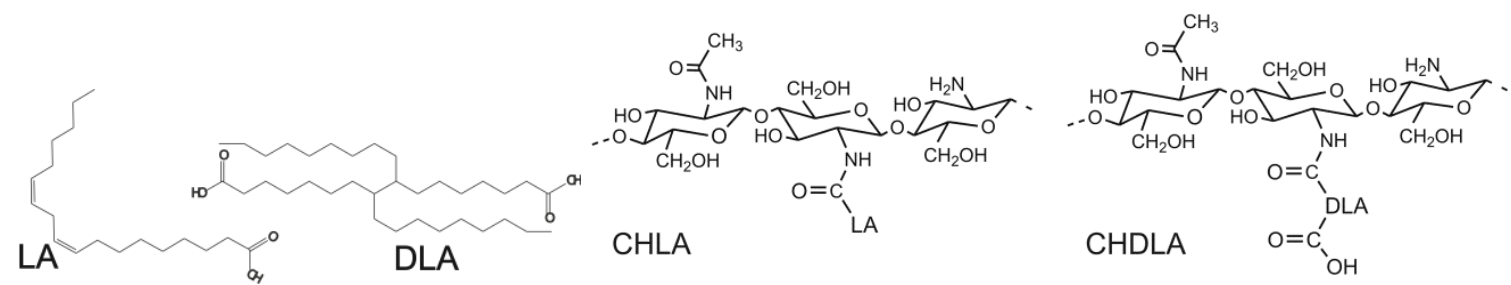

Fig. 5. Chemical structure of linoleic and dilinoleic fatty acids and chitosan-fatty acid derivatives. 


\subsubsection{Commercial Coatings}

As reference materials, three different commercial coatings were investigated, all of which were hydrophilic and medical grade, see table 1 . The first coating (C1) was a non-toxic thermoplastic hydrophilic copolymer based on vinylpyrrolidone and acrylate block, commonly used as a coating for medical devices such as guide wires and catheters. Coatings $\mathrm{C} 2$ and $\mathrm{C} 3$ were ether-based hydrophilic urethanes designed for use as coatings for a wide range of medical devices. According to data from the supplier, C2 has 50\% water absorption and 50\% linear expansion and C3 has 90\% water absorption and $100 \%$ linear expansion.

Table 1. Summary of coating materials.

\begin{tabular}{|l|l|l|}
\hline Designation & Type of polymer & Comments \\
\hline CH & Polysaccharide & chitosan \\
\hline CHLA & Polysaccharide/fatty acid & chitosan/linoleic acid \\
\hline CHDLA & Polysaccharide/fatty acid & chitosan/dilinoleic acid \\
\hline C1 & Copolymer & vinylpyrrolidone and acrylate copolymer \\
\hline C2 & Ether-based hydrophilic urethane & $50 \%$ water absorption, 50\% linear expansion \\
\hline C3 & Ether-based hydrophilic urethane & $90 \%$ water absorption, 100\% linear expansion \\
\hline
\end{tabular}

\subsubsection{Coatings preparation}

Coatings from chitosan and chitosan derivatives were prepared by dip-coating on activated PA probes. The activation of the surfaces was carried out by immersing the probes in $30 \%$ hydrogen peroxide for 30 minutes at $70^{\circ} \mathrm{C}$ and then in EDC/alcohol solution for $30 \mathrm{~min}$ at room temperature. The catalyst solution - EDC/alcohol solution was obtained by dissolving 1g of 1-ethyl-3-(3-dimethylaminopropyl) carbodiimide $(E D C)$ in a mixture of ethanol $(10 \mathrm{ml})$ and isopropanol $(40 \mathrm{ml})$. Activated PA probes were dipped into a chitosan or chitosan derivatives solution ( $2 \%$ wt. in $1 / 1$ mixture of $1 \%$ acetic acid/isopropanol $(\mathrm{v} / \mathrm{v})$ ), held for $2 \mathrm{~min}$ and then dried in air.

The commercial coatings were prepared according to the manufacturer's instructions, also using the dip-coating technique. The polymer probes were dipped in 5\% (wt.) coating solutions in 1-methyl-2pyrrolidone (NMP) for $2 \mathrm{~min}$. The probes were then dried for $2.5 \mathrm{~h}$ at $55^{\circ} \mathrm{C}$.

\subsection{Contact angle measurement}

Contact angle measurements were carried out on the coatings because this parameter is an indicator of the surface properties (hydrophilic or hydrophobic), which are important for the lubricity. The water contact angle was measured by sessile drop shape analysis according to European Standard EN 828 using a goniometer Krüss DSA 100 Drop Shape Analyzer equipped with a camera and recording system. Contact angle test samples were prepared by dip coating PA disks, in the same way as for the tribological test probes. A $2-\mu \mathrm{L}$ distilled water drop was applied on different areas of the sample. The contact angle was measured every second for a period of 60 seconds. Contact angle values for 1,15 , 30 , and 60 seconds were selected as measurement points. The average angle was calculated from 5 measurements. Additional measurements were performed with coated PA disks immersed in blood analogue for $30 \mathrm{~min}$, followed by blotting to remove surface moisture.

\section{Results and discussion}

\subsection{Friction behaviour of uncoated polymer}

Fig. 6 shows the average change in coefficient of friction (COF) with time (at a sliding velocity 0.5 $\mathrm{mm} / \mathrm{s}$ ) for the uncoated polyamide probes, PA33 and PA69. The error bars in this figure represent one standard deviation (taken from five repeat tests). The CoF values for both materials begin at around 0.2. However, whilst the CoF of the harder PA69 material remained almost constant for the duration of the test, the CoF of the softer PA33 material gradually increased with sliding distance. The reason for this increase is unclear; however, it may be attributed to significant plastic deformation of the 
softer material occurring during the test, resulting in a gradually increasing contact surface area in time and hence greater adhesion and/or deformation friction.

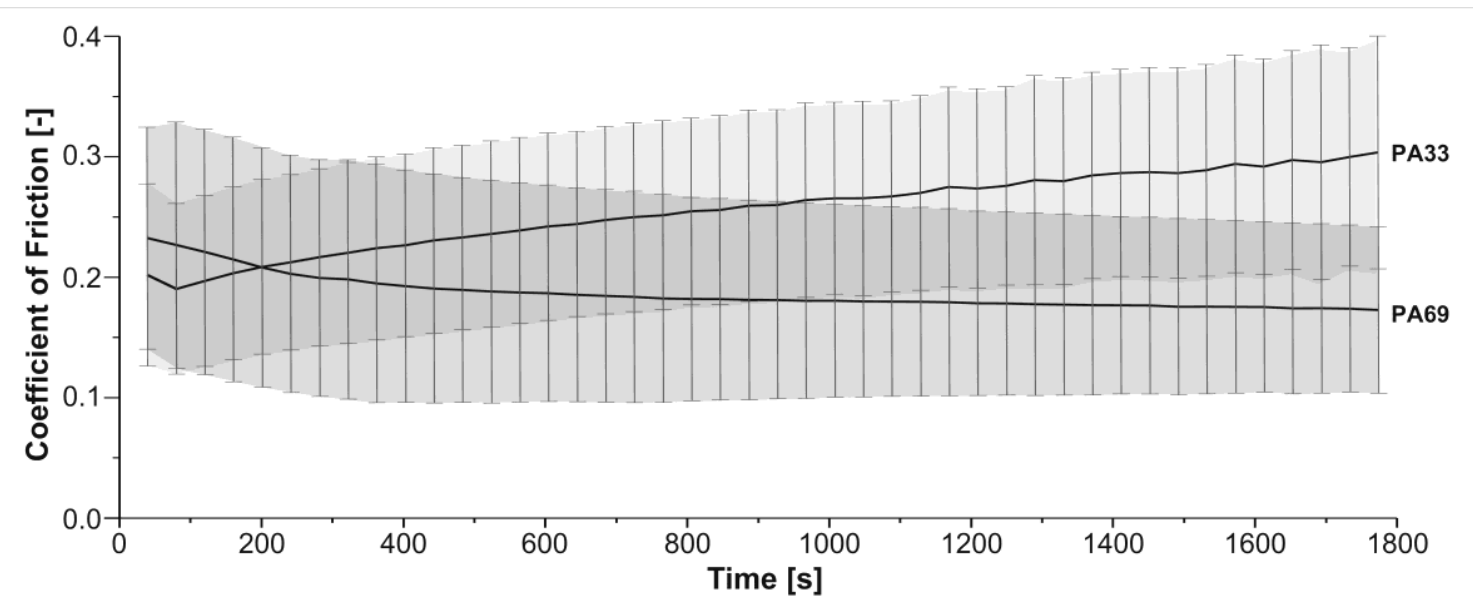

Fig. 6 Measured average coefficient of friction for uncoated polyamides PA33 and PA69. The error bars represent one standard deviation.

\subsection{Friction behaviour of coatings}

Fig. 7 shows a comparison of the average friction behaviour of the uncoated polyamides (PA33, PA69) and the same material coated by chitosan $(\mathrm{CH})$ and the chitosan derivatives (CHLA and CHDLA) (panels $A$ and $C$ ), as well as commercial coatings (panels B and D).
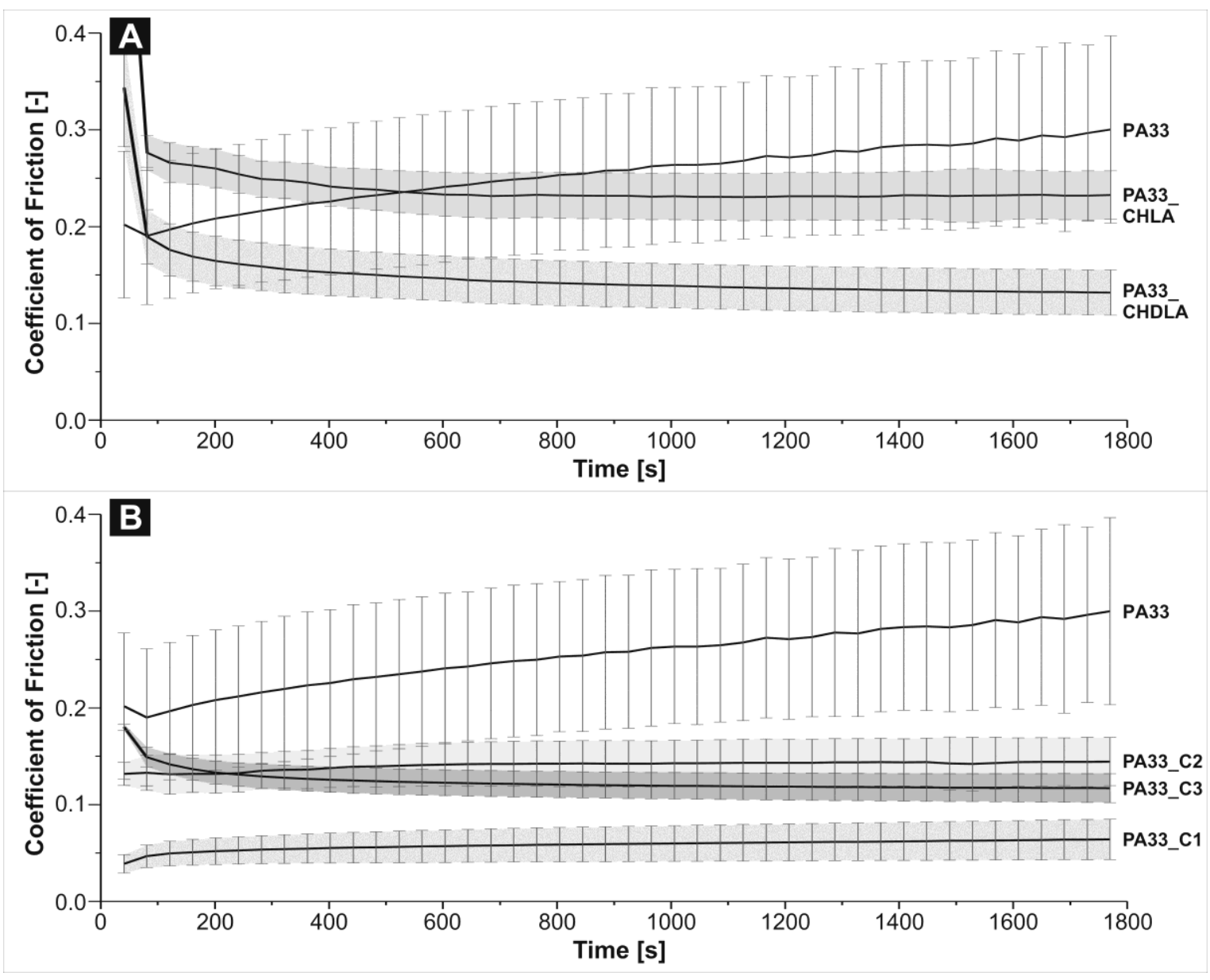

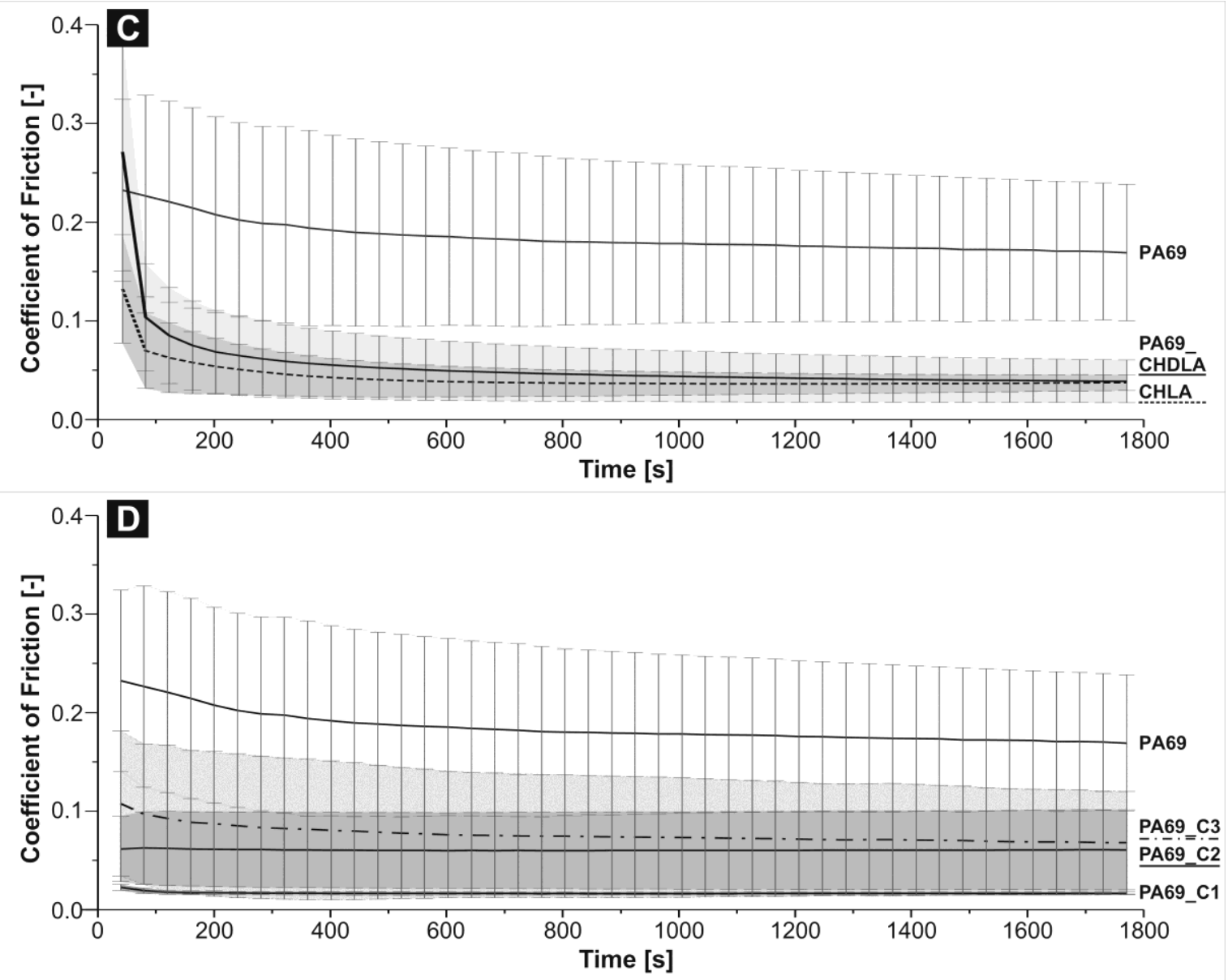

Fig. 7 Average coefficient of friction as a function of time for PA uncoated and coated by chitosan and its derivatives or commercial coatings. Panels A and B represent PA33. Panels C and D represent PA69.

With all the coatings tested, the friction coefficient was initially relatively high but decreased during a short running-in period to a lower, stable value or remained stable for the remaining duration of the test. An increase in the friction during this period, becoming closer to the value for uncoated material, would have been an indication of coating wear and insufficient lifetime. No such increase in the friction coefficient was observed during the test period for any of the coatings tested, indicating that all of the coatings had sufficient lifetime as regards wear performance.

It is clear that all of chitosan coatings show lower CoF than the uncoated material, demonstrating the lubricious properties of chitosan itself. It is interesting to note that there are very few references in the literature to research on the friction reducing effect of chitosan $[44,45]$. The current work is the first in which chitosan and chitosan derivatives have been applied as lubricious coatings. After running-in, both of the chitosan derivatives tested showed approximately the same CoF behaviour with the harder (PA69) polymer material as substrate, indicating that the type of fatty acid used in the chitosan derivative did not have a significant influence.

The chitosan derivative coatings were also compared with three hydrophilic commercial coatings, see Table 1. Fig $8 A$ and $B$ show the average CoF values for the uncoated and coated soft PA33 (A) and hard PA69 (B) materials, respectively. For each test, the CoF values shown in Fig. 8 are averages from the test period between 800 and 1700 s sliding time, when the CoF had reached a relatively stable and constant value following the initial running-in period. 


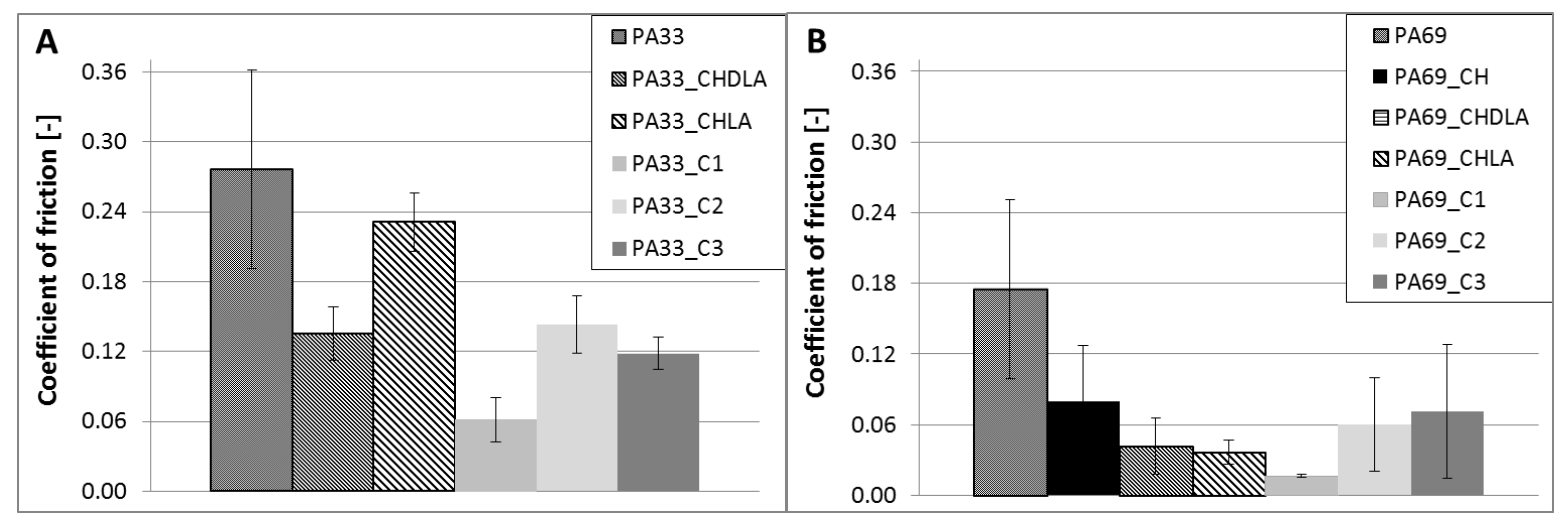

Fig. 8 Average coefficient of friction results for all tests using (A) softer PA33 polymer and (B) harder PA69 polymer substrates for the coatings. The errors bars indicate one standard deviation derived from at least five separate tests, each using fresh materials and coatings.

Fig. 8 shows that all of the coatings tested decreased the coefficient of friction compared to the uncoated polymer but that there are differences between the results for the softer PA33 and harder PA69 substrates. It has already been mentioned that, in general, the higher observed friction values for the PA33 material may be attributed to greater viscoelastic deformation of this softer material, leading to a greater contact area and hence greater adhesion and/or deformation friction. In addition, the Pebax substrate used is a block copolymer, where the phase structure and bulk mechanical properties, such as hardness, depend on the relative amounts of its "hard" and "soft" constituents (blocks). The two substrate polymers have different block compositions, with PA33 having more hydrophilic ether "soft" blocks. This may affect the interaction between the amphiphilic coating molecules and the substrate. This effect may play a greater role in the case LA-modified chitosan, due to LA having a lower molecular weight and being more able to reorganize. We hypothesized that these interactions would have an effect on the contact angle and water absorption properties of the applied coatings. This in turn, would then be expected to influence the friction behaviour of the coatings. Importantly, in the case of the coated PA69 polymer, which is representative of the bulk of the length of a guide catheter, it is clear that both of the chitosan derivatives coatings (CHLA and CHDLA) reduced the friction to levels similar to those of the commercially-available coatings $\mathrm{C} 1, \mathrm{C} 2$ and $\mathrm{C} 3$.

\subsection{Surface wettability}

The wettability of biomaterials is very important for their biocompatibility, especially hemocompatibility [46] and this also can influence the friction behaviour [47]. In general, hydrophilic surfaces provide good lubricity while surface hydrophobicity can lead to thrombogenesis arising from preferred fibrinogen adsorption activating adhesion of platelets and clotting [48].

In order to explain the time course of friction behaviour, the hydrophilicity of the chitosan coatings was characterized by measurement of the water contact angle every second for a period of 60 seconds. A typical result is shown in Fig. 9 for the CHDLA coating after 60s.

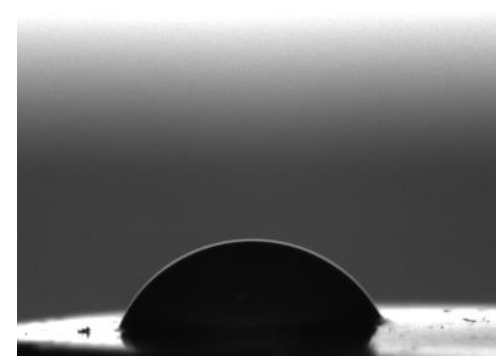

Fig. 9 Sessile water drop on CHDLA coating, after 60s. 

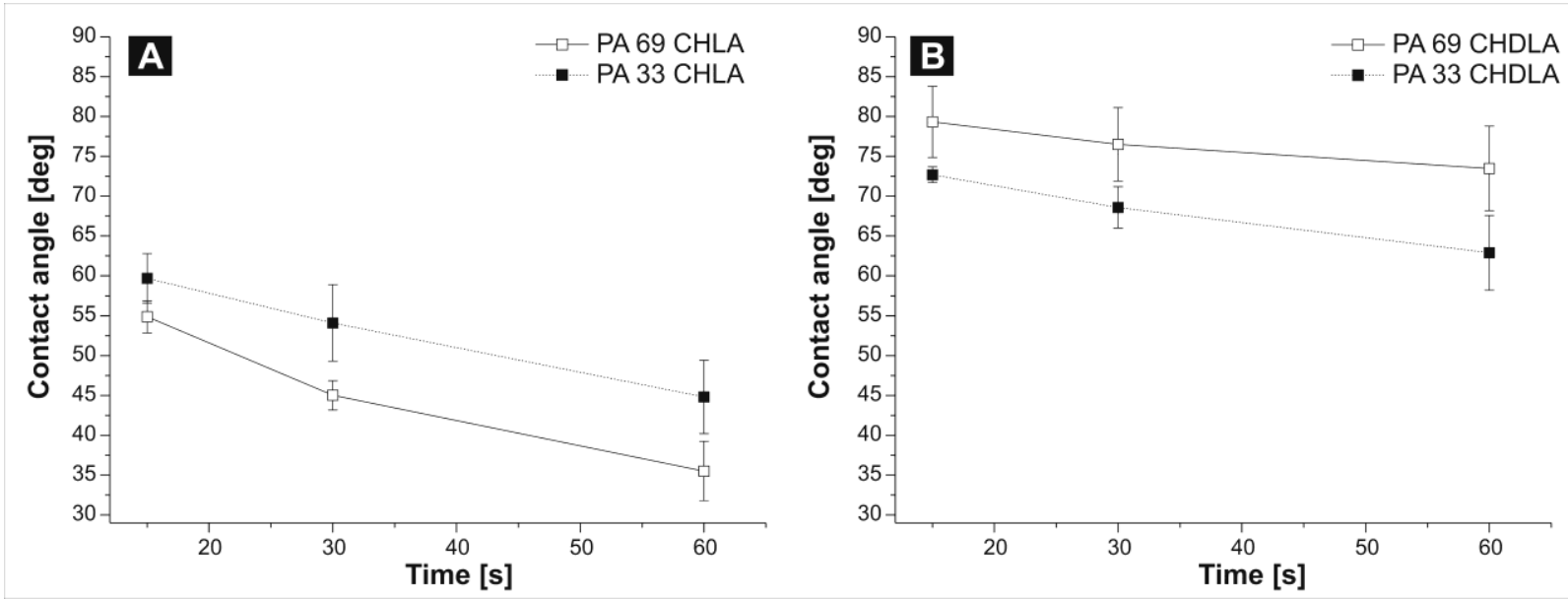

Fig 10. Time course of contact angle measurements for CHLA (a) and CHDLA (b) coatings on PA.

The results are presented in Figure 10, which shows averages from five separate measurements at each time point. By comparison, for all contact times up to 60s, the two uncoated polyamides exhibited angles of $90^{\circ}$ (PA33) and $101^{\circ}$ (PA69), respectively, typical for hydrophobic surfaces, while unmodified chitosan $(\mathrm{CH})$ exhibited contact angle of $34^{\circ}$, indicating its hydrophilic character.

Modification of the chitosan by the hydrophobic fatty acid compounds (monocarboxylic LA and dicarboxylic DLA) results in an increase of the contact angle compared to $\mathrm{CH}$. Overall, $\mathrm{CHLA}$ has a lower contact angle (about $50-60^{\circ}$ after $15 \mathrm{~s}$ ) than CHDLA (about $75-80^{\circ}$ after $15 \mathrm{~s}$ ). Additionally, we observe a difference in behaviour between the two coatings on the different substrates. On PA33, CHLA has a higher contact angle than on PA69 (Fig. 10a), while the opposite is true for CHDLA (Fig. 10b). These differences may relate to structural differences between the derivatives relative to the substrate. PA33 has more hydrophilic ether "soft" segments, which may favor greater interaction with the hydrophilic chitosan chains while the coating forms, as compared to PA69. Thus, for the case of CHLA, having shorter pendant aliphatic chain, which is able to reorganize more readily, the outer surface of the coating will tend to have more LA groups, resulting in a higher contact angle, as compared to CHLA on PA69. PA69, which has less ether blocks, will tend allow for more chitosan chains to be exposed on the surface, compared to PA33. In the case of CHDLA, the DLA fatty acid tail is terminated with an additional carboxylic $(-\mathrm{COOH})$ group (see Fig. 5$)$ and this may favour interactions with the ether blocks of PA33. Additionally, it is possible that via this second carboxylic group, some DLA may react two different chitosan molecules, acting as a crosslinker, reducing the polymer chain mobility, but potentially increasing the surface energy. On PA33 (Fig. 10b), this may expose more chitosan on the surface, resulting in lower contact angle, as compared to PA69.

Upon exposure to aqueous environment, the surface molecules of the coatings will rearrange in order to minimize interface energy, exposing more hydrophilic chitosan over time. This effect will result in the reduction of contact angle, as observed for both coating materials (Fig. 10). Such a rearrangement could lead to alignment of the molecules in the sliding direction and result in a decreased friction coefficient. However, the difference in time course between the two coatings is likely due to the fact that DLA will tend to hinder rearrangement.

Additionally, during the friction experiments, as shown in Figure 2, the probe was partially immersed in the glycerol-water mixture to a (consistent) depth of approximately $3 \mathrm{~mm}$. A change in contact angle between the liquid and the coating on the probe will also result in a change in surface tension at meniscus formed between the fluid and the probe. This change in normal force on the probe is not taken into account in the calculation of the friction coefficient and may result in imprecision. This force may also further encourage a boundary lubrication condition. Further studies will be needed to address the mechanism of time-dependent reduction of CoF and contact angle for the chitosan-fatty 
acid coatings[49], including careful measurement of the forces acting vertically on the coated probes as they approach and become immersed in the fluid before starting the friction test.

However, after 30 min exposure to the aqueous solution, both coatings exhibit zero contact angles, indicating an equilibrium condition of complete saturation with water, potentially accounting for similar final CoF by the formation of a lubrication layer.

\section{Conclusions}

Our work here presents the development of an in vitro tribological system for testing catheter coatings, intended to represent, to a limited extent, the in vivo friction conditions of a typical endovascular catheterization procedure. Using the developed tribological system, we tested the friction behavior of novel modified chitosan coatings and compared them to several commercially available coatings. To our knowledge, this is the first time such tests have been performed. Our results indicate that chitosan and chitosan modified with linoleic or dilinoleic acid acts as a lubricious coating, markedly reducing the coefficient of friction, as compared to neat polyamide substrate. In fact, the chitosan-fatty acid coatings were comparable to hydrogel commercial coatings in terms of the coefficient of friction, while providing the advantages of being biodegradable and antimicrobial. Our results highlight the potential of these novel coatings for both intravascular, as well as urinary, catheter applications, where lubricious coatings combined with antimicrobial activity in particular would be highly desired.

\section{Acknowledgements}

This project has received funding from the European Union's Seventh Framework Programme for research, technological development and demonstration under grant agreement no. 286174 "UNITISS". Partial financial support from the National Science Centre grant N N507 319440 (chitosan derivative synthesis) is also acknowledged. We thank G. Lutz, PhD, A. Piegat, PhD and P. Sobolewski, $\mathrm{PhD}$, for helpful discussions and editing of the manuscript.

\section{References}

[1] Tan A, Farhatnia Y, de Mel A, Rajadas J, Alavijeh MS, Seifalian AM. Inception to actualization: Next generation coronary stent coatings incorporating nanotechnology. J Biotechnol 2013;164:170-151. doi:10.1016/j.jbiotec.2013.01.020.

[2] Trajkovski B, Petersen A, Strube P, Mehta M, Duda GN. Intra-operatively customized implant coating strategies for local and controlled drug delivery to bone. Adv Drug Deliv Rev 2012;64:1142-51. doi:10.1016/j.addr.2012.05.016.

[3] Guo L, Yuan W, Lu Z, Li CM. Polymer/nanosilver composite coatings for antibacterial applications. Colloids Surfaces A Physicochem Eng Asp 2013;439:69-83. doi:10.1016/j.colsurfa.2012.12.029.

[4] Hosseini S, Ibrahim F, Djordjevic I, Koole LH. Polymethyl methacrylate-co-methacrylic acid coatings with controllable concentration of surface carboxyl groups: A novel approach in fabrication of polymeric platforms for potential bio-diagnostic devices. Appl Surf Sci 2014;300:43-50. doi:10.1016/j.apsusc.2014.01.203.

[5] Bax D V, Kondyurin A, Waterhouse A, McKenzie DR, Weiss AS, Bilek MMM. Surface plasma modification and tropoelastin coating of a polyurethane co-polymer for enhanced cell attachment and reduced thrombogenicity. Biomaterials 2014;35:6797-809.

doi:10.1016/j.biomaterials.2014.04.082. 
[6] Sobolewski P, El Fray M. Cardiac catheterization: consequences for the endothelium and potential for nanomedicine. WIREs Nanomedicine and Nanobiotechnology 2014; doi: 10.1002/wnan.1316.

[7] Dellimore KH, Franklin SE, Helyer AR. A Review of Catheter Related Complications During Minimally Invasive Transcatheter Cardiovascular Intervention with Implications for Catheter Design. Cardiovasc Eng Technol 2014. doi:10.1007/s13239-014-0183-9.

[8] Wyman P. Coatings for Biomedical Applications. Elsevier; 2012. doi:10.1533/9780857093677.1.3.

[9] Dawson EA, Rathore S, Cable NT, Wright DJ, Morris JL, Green DJ. Impact of introducer sheath coating on endothelial function in humans after transradial coronary procedures. Circ Cardiovasc Interv 2010;3:148-56. doi:10.1161/CIRCINTERVENTIONS.109.912022.

[10] Rathore S, Stables RH, Pauriah M, Hakeem A, Mills JD, Palmer ND, et al. Impact of length and hydrophilic coating of the introducer sheath on radial artery spasm during transradial coronary intervention: a randomized study. JACC Cardiovasc Interv 2010;3:475-83. doi:10.1016/j.jcin.2010.03.009.

[11] Caussin C, Gharbi M, Durier C, Ghostine S, Pesenti-Rossi D, Rahal S, et al. Reduction in spasm with a long hydrophylic transradial sheath. Catheter Cardiovasc Interv 2010;76:668-72. doi:10.1002/ccd.22552.

[12] Saririan M, Pyne CT. Sterile granuloma formation following radial artery catheterization: too many Cooks? Catheter Cardiovasc Interv 2010;76:907-8. doi:10.1002/ccd.22357.

[13] Gilchrist IC, Kozak M. Hydrophilic-coated radial sheaths: a leap forward, but watch where you land. JACC Cardiovasc Interv 2010;3:484-5. doi:10.1016/j.jcin.2010.03.008.

[14] Subramanian R, White CJ, Sternbergh WC, Ferguson DL, Gilchrist IC. Nonhealing wound resulting from a foreign-body reaction to a radial arterial sheath. Catheter Cardiovasc Interv 2003;59:205-6. doi:10.1002/ccd.10468.

[15] Kozak M, Adams DR, loffreda MD, Nickolaus MJ, Seery TJ, Chambers CE, et al. Sterile inflammation associated with transradial catheterization and hydrophilic sheaths. Catheter Cardiovasc Interv 2003;59:207-13. doi:10.1002/ccd.10522.

[16] Ziakas A, Karkavelas G, Mochlas S. Sterile inflammation after transradial catheterization using a hydrophilic sheath: a case report. Int J Cardiol 2005;99:495-6.

doi:10.1016/j.ijcard.2004.01.047.

[17] Sado D, Witherow F. Sterile granuloma formation following radial artery facilitated coronary catheterization. Catheter Cardiovasc Interv 2009;74:606. doi:10.1002/ccd.22048.

[18] Zellner C, Yeghiazarians Y, Ports TA, Ursell P, Boyle AJ. Sterile radial artery granuloma after transradial cardiac catheterization. Cardiovasc Revascularization Med 2011;12:187-9. doi:10.1016/j.carrev.2010.06.003.

[19] Harish Prashanth KV, Tharanathan RN. Chitin/chitosan: modifications and their unlimited application potential-an overview. Trends Food Sci Technol 2007;18:117-31.

doi:10.1016/j.tifs.2006.10.022. 
[20] Muzzarelli R. Chitins and chitosans for the repair of wounded skin, nerve, cartilage and bone. Carbohydr Polym 2009;76:167-82. doi:10.1016/j.carbpol.2008.11.002.

[21] Dash M, Chiellini F, Ottenbrite RM, Chiellini E. Chitosan-A versatile semi-synthetic polymer in biomedical applications. Prog Polym Sci 2011;36:981-1014.

doi:10.1016/j.progpolymsci.2011.02.001.

[22] Elsabee MZ, Morsi RE, Al-Sabagh AM. Surface active properties of chitosan and its derivatives. Colloids Surf B Biointerfaces 2009;74:1-16. doi:10.1016/j.colsurfb.2009.06.021.

[23] Aranaz I, Harris R, Heras A. Chitosan amphiphilic derivatives. Chemistry and applications. Curr Org Chem 2010;14:308-30.

[24] Cai G, Jiang H. pH-sensitive nanoparticles self-assembled from a novel class of biodegradable amphiphilic copolymers based on chitosan. J Mater Sci Mater Med 2009;20:1315-20. doi:10.1007/s10856-008-3689-6.

[25] Vargas M, Albors A, Chiralt A, González-Martínez C. Characterization of chitosan-oleic acid composite films. Food Hydrocoll 2009;23:536-47. doi:10.1016/j.foodhyd.2008.02.009.

[26] Balan V, Verestiuc L. Strategies to improve chitosan hemocompatibility: A review. Eur Polym J 2014;53:171-88. doi:10.1016/j.eurpolymj.2014.01.033.

[27] El Fray M, Niemczyk A, Pabin-Szafko B. Chemical Modification of Chitosan with Fatty Acids. Prog Chem Appl Chitin Its Deriv 2012;XVII:29-36.

[28] Niemczyk A, Fray M El. NOVEL CHITOSAN DERIVATIVES AS FILMS WITH AN ANTIMICROBIAL EFFECT. Prog Chem Appl Chitin Its Deriv 2013;XVIII:59-66.

[29] Raad I, Hanna H, Maki D. Intravascular catheter-related infections: advances in diagnosis, prevention, and management. Lancet Infect Dis 2007;7:645-57. doi:10.1016/S14733099(07)70235-9.

[30] Hooton TM, Bradley SF, Cardenas DD, Colgan R, Geerlings SE, Rice JC, et al. Diagnosis, Prevention, and Treatment of Catheter-Associated Urinary Tract Infection in Adults: 2009 International Clinical Practice Guidelines from the Infectious Diseases Society of America. Clin Infect Dis 2010;50:625-63. doi:10.1086/650482.

[31] Lee J-H, Lee S-S, Chang J-D, Thompson MS, Kang D-J, Park S, et al. A novel method for the accurate evaluation of Poisson's ratio of soft polymer materials. Sci World J 2013;2013:930798. doi:10.1155/2013/930798.

[32] Ohta $M$, Handa $A$, Iwata $H$, Rüfenacht $D$, Tsutsumi S. Poly-vinyl alcohol hydrogel vascular models for in vitro aneurysm simulations: the key to low friction surfaces. Technol Health Care 2004;12:225-33.

[33] Yu C, Kosukegawa H, Mamada K, Kuroki K, Takashima K, Yoshinaka K, et al. Development of an In Vitro Tracking System with Poly (vinyl alcohol) Hydrogel for Catheter Motion. J Biomech Sci Eng 2010;5:11-7. doi:10.1299/jbse.5.11. 
[34] Kosukegawa H, Mamada K, Kuroki K, Liu L, Inoue K, Hayase T, et al. Measurements of Dynamic Viscoelasticity of Poly (vinyl alcohol) Hydrogel for the Development of Blood Vessel Biomodeling. J Fluid Sci Technol 2008;3:533-43. doi:10.1299/jfst.3.533.

[35] Mansur HS, Sadahira CM, Souza AN, Mansur AAP. FTIR spectroscopy characterization of poly (vinyl alcohol) hydrogel with different hydrolysis degree and chemically crosslinked with glutaraldehyde. Mater Sci Eng C 2008;28:539-48. doi:10.1016/j.msec.2007.10.088.

[36] Watler PK, Cholakis CH, Sefton M V. Water content and compression modulus of some heparin-PVA hydrogels. Biomaterials 1988;9:150-4. doi:10.1016/0142-9612(88)90114-7.

[37] Mamada K, Fridrici V, Kosukegawa H, Kapsa P, Ohta M. Friction Properties of Poly(vinyl alcohol) Hydrogel: Effects of Degree of Polymerization and Saponification Value. Tribol Lett 2011;42:241-51. doi:10.1007/s11249-011-9768-8.

[38] Mamada K, Kosukegawa H, Fridrici V, Kapsa P, Ohta M. Friction properties of PVA-H/steel ball contact under water lubrication conditions. Tribol Int 2011;44:757-63. doi:10.1016/j.triboint.2010.12.014.

[39] Campo-Deaño L, Dullens RP a, Aarts DG a L, Pinho FT, Oliveira MSN. Viscoelasticity of blood and viscoelastic blood analogues for use in polydymethylsiloxane in vitro models of the circulatory system. Biomicrofluidics 2013;7:34102. doi:10.1063/1.4804649.

[40] Wickramasinghe SR, Kahr CM, Han B. Mass transfer in blood oxygenators using blood analogue fluids. Biotechnol Prog 2002;18:867-73. doi:10.1021/bp010192h.

[41] Yousif MY, Holdsworth DW, Poepping TL. A blood-mimicking fluid for particle image velocimetry with silicone vascular models. Exp Fluids 2010;50:769-74. doi:10.1007/s00348010-0958-1.

[42] De Simone G, Devereux RB, Chinali M, Best LG, Lee ET, Welty TK. Association of blood pressure with blood viscosity in american indians: the Strong Heart Study. Hypertension 2005;45:62530. doi:10.1161/01.HYP.0000157526.07977.ec.

[43] Dintenfass L. Blood Viscosity. Springer Science \& Business Media; 1985.

[44] Arizmendi-Morquecho a., Chávez-Valdez a., Navarro CH, Moreno KJ. Performance evaluation of chitosan/hydroxyapatite composite coating on ultrahigh molecular weight polyethylene. Polym Test 2013;32:32-7. doi:10.1016/j.polymertesting.2012.10.002.

[45] Pettersson T, Dedinaite A. Normal and friction forces between mucin and mucin-chitosan layers in absence and presence of SDS. J Colloid Interface Sci 2008;324:246-56. doi:10.1016/j.jcis.2008.04.055.

[46] Chen KY, Kuo JF, Chen CY. Synthesis, characterization and platelet adhesion studies of novel ion-containing aliphatic polyurethanes. Biomaterials 2000;21:161-71.

[47] Ma X, Ding G, Zhang Y, Wang K. Effects of hydrophilic coating on air side heat transfer and friction characteristics of wavy fin and tube heat exchangers under dehumidifying conditions. Energy Convers Manag 2007;48:2525-32. doi:10.1016/j.enconman.2007.03.017. 
[48] Yuan W, Feng $Y$, Wang H, Yang D, An B, Zhang W, et al. Hemocompatible surface of electrospun nanofibrous scaffolds by ATRP modification. Mater Sci Eng C Mater Biol Appl 2013;33:3644-51. doi:10.1016/j.msec.2013.04.048.

[49] Kleingartner J, Lee H, Rubner MF, McKinley GH, Cohen RE. Exploring the kinetics of switchable polymer surfaces with dynamic tensiometry. Soft Matter 2013;9:6080. doi:10.1039/c3sm50596k. 